\title{
A consistent extension of the local spin density approximation to account for quantum dot mass and dielectric mismatches
}

\author{
M. Pi \\ Departament ECM, Facultat de Fýsica and $I N^{2}$ UB, Universitat de Barcelona, E-08028 Barcelona, Spain \\ M. Royo and J. Planelles ${ }^{\text {a) }}$ \\ Departament de Ciències Experimentals, Universitat Jaume I, Box 224, E-12080 Castelló, Spain
}

(Received 12 May 2006; accepted 18 July 2006; published online 12 October 2006)

\begin{abstract}
A consistent extension of local spin density approximation (LSDA) to account for mass and dielectric mismatches in nanocrystals is presented. The extension accounting for variable effective mass is exact. Illustrative comparisons with available configuration interaction calculations show that the approach is also very reliable when it comes to account for dielectric mismatches. The modified LSDA is as fast and computationally low demanding as LSDA. Therefore, it is a tool suitable to study large particle systems in inhomogeneous media without much effort. (C) 2006 American Institute of Physics. [DOI: 10.1063/1.2356791]
\end{abstract}

\section{INTRODUCTION}

Quantum dot (QD) devices where the Coulomb interaction is exploited to control charge injection at the singleelectron level have been achieved in recent years. ${ }^{1,2}$ This has a huge technological interest. For example, the operation of QD memory devices basically consists of storing (programming), holding (retention), and removing (erase) charges in the QD by means of the device gate voltage. ${ }^{3}$ The importance of the single-electron transistors relies on the extremely low power required for their operation (of the order of nanowatts). A key quantity that characterizes transport into a QD is the addition energy, i.e., the energy $E_{\text {add }}(N)$ required in order to place an extra electron into a dot that is initially occupied by $N-1$ particles. Such quantity, analogous to electron affinity in atomic physics, can be measured experimentally as a function of $N$. Thus, when the conductance is measured as a function of an applied gate voltage $V_{g}$, a series of conductance peaks appears. Each peak corresponds to an additional electron in the dot, and the spacings between the conductance peaks $\Delta V_{g}$ are proportional to the change in the chemical potential of the dot as an additional electron enters. This conductance peak spacing is mainly determined by the charging energy and it is known as Coulomb blockade. ${ }^{4}$ The possibility to measure addition spectra of quantum dots by single-electron capacitance ${ }^{5}$ or transport spectroscopy ${ }^{6}$ stimulated many ground-state calculations. The most rigorous, and computationally very demanding, exact diagonalization can only be applied to few-electron systems. Larger systems require less-demanding methods, such as the density functional theory (DFT). The practical limitations of this method comes from the not exactly known exchangecorrelation potential, but general experience is that DFT results are quite reliable ${ }^{7}$ and they have contributed substantially to an understanding of quantum dot addition spectra. ${ }^{8,9}$

As pointed out above, single-electron transistors require extremely low power for their operation. This opens the way

${ }^{\text {a)} E l e c t r o n i c ~ m a i l: ~ j o s e p . p l a n e l l e s @ e x p . u j i . e s ~}$ to their possible integration in bioenvironments. The integration in these environments is generally incompatible with the large power dissipation of current microelectronic transistors, which are orders of magnitude larger. Colloidal chemistry techniques allow QDs to be synthesized in the form of semiconductor spherical nanocrystals, with very low defect densities and size dispersion. These QDs can also be fabricated as multishell structures, ${ }^{10}$ i.e., built of concentric layers (shells) of different semiconductors with the shell thickness down to a single monolayer. The size of the nanocrystals and the composition of layers can be easily manipulated in the process of fabrication, which makes it possible to tailor to a large extent their discrete energy spectra. ${ }^{11}$ These QDs can be embedded in various kinds of matrices, such as glasses ${ }^{12}$ or organic and biological materials ${ }^{13}$ being very promising for applications, in particular, for the integration of nanoelectronic devices in biological environments. ${ }^{14}$ It has been also shown that these QDs embedded in low-dielectric-constant matrices can be built into single-electron transistors ${ }^{1,14}$ and that capacitance or tunneling spectroscopies can be used to obtain their addition spectra. ${ }^{15-17}$ A specific characteristic of organic environments is their huge dielectric mismatch with typical inorganic semiconductor QD structures. When QDs are embedded in such materials, the formation of polarization charges at the interface may strongly influence confinement and charging energies and modify the distribution of charge carriers inside the QD. Therefore, the effects of the large mass and dielectric mismatches cannot be overlooked in the interpretation of single-electron charging phenomena in these dots. These effects have been incorporated for spherical few-electron QDs by employing diagonalization procedures within a Hubbard-like approximation, whereby only the semidiagonal elements of the Hamiltonian matrix are retained. ${ }^{18,19}$ Some attempts to incorporate these effects in the local density approximation (LDA), which allows to deal with large systems, have also been reported. ${ }^{20,21}$ In the present paper we extend, in a consistent way, the successful local spin density approximation (LSDA) to account for quantum dot mass and dielectric mismatches. The paper is 
organized as follows: the next section presents the formulation of the method. Next, illustrative comparisons with more sophisticated calculations available are carried out, and some concluding remarks end the paper.

\section{THEORY}

\section{A. Outline of density functional theory}

Density functional theory ${ }^{22,23}$ (DFT) in the selfconsistent formulation of Kohn and $\mathrm{Sham}^{24}$ has proven to be a particularly powerful tool to study large electron systems in the presence of correlation. According to Hohenberg and $\mathrm{Kohn}^{25}$ and its generalization by Levy, ${ }^{26}$ the exact groundstate energy of a many-body system is a unique functional of the electron density $n(\mathbf{r})$. Although DFT was initially developed in a spin-independent formalism, effects of spin polarization were later incorporated in the so-called spin density functional theory (SDFT). ${ }^{27}$ In this approach the total energy is a functional of the spin-up and spin-down densities $n_{\sigma}(\mathbf{r})$, where $\sigma=(+,-)$ labels the spin. Equivalently, the energy is a functional of the total density $n(\mathbf{r})=n_{+}(\mathbf{r})+n_{-}(\mathbf{r})$ and spin polarization $\zeta(\mathbf{r})=\left[n_{+}(\mathbf{r})-n_{-}(\mathbf{r})\right] / n(\mathbf{r})$. The ground state is found by minimizing the energy functional, leading to the well known Kohn-Sham equations,

$$
\left[-\frac{\hbar^{2}}{2 m} \nabla^{2}+V_{\mathrm{KS}, \sigma}(n, \zeta)\right] \Phi_{i \sigma}(\mathbf{r})=E_{i \sigma} \Phi_{i \sigma}(\mathbf{r}),
$$

with

$$
V_{\mathrm{KS}, \sigma}(n, \zeta)=V_{\mathrm{conf}}(\mathbf{r})+\int d \mathbf{r}^{\prime} \frac{n\left(\mathbf{r}^{\prime}\right)}{\left|\mathbf{r}-\mathbf{r}^{\prime}\right|}+\frac{\delta E_{\mathrm{xc}}(n, \zeta)}{\delta n_{\sigma}(\mathbf{r})},
$$

the last term being the exchange-correlation contribution. ${ }^{28}$

For a finite system with nonuniform density $n(\mathbf{r})$, the assumption that, locally, the exchange-correlation energy can be obtained by applying uniform-electron-gas results $e_{\mathrm{xc}}[n(\mathbf{r}), \zeta(\mathbf{r})]$ to infinitesimal portions of the nonuniform electron distribution is commonly made:

$$
E_{\mathrm{xc}}(n, \zeta)=\int d \mathbf{r} n(\mathbf{r}) e_{\mathrm{xc}}[n(\mathbf{r}), \zeta(\mathbf{r})]
$$

The functional $E_{\mathrm{xc}}(n, \zeta)$ can in turn be divided into exchange and correlation contributions,

$$
E_{\mathrm{xc}}(n, \zeta)=E_{x}(n, \zeta)+E_{c}(n, \zeta) .
$$

In the local approximation, the exchange part is given by the Dirac exchange energy functional, corresponding to a homogeneous electron gas,

$$
E_{x}(n, \zeta)=\frac{C_{x}}{2} \int d \mathbf{r} n(\mathbf{r})^{4 / 3}\left\{[1+\zeta(\mathbf{r})]^{4 / 3}+[1-\zeta(\mathbf{r})]^{4 / 3}\right\},
$$

where $C_{x}=\frac{3}{4}(3 / \pi)^{1 / 3}$. It can be rewritten as

$$
E_{x}(n, \zeta)=\int d \mathbf{r} n(\mathbf{r}) \epsilon_{x}(n, \zeta)
$$

All approximations introduced so far should be compensated by a proper selection of the correlation term $E_{c}(n, \zeta)$. In the local approximation,

$$
E_{c}(n, \zeta)=\int d \mathbf{r} n(\mathbf{r}) \epsilon_{c}(n, \zeta)
$$

There are different parametrizations for this term. In our calculations we have selected the commonly employed Perdew and Zunger functional, ${ }^{29}$ which is expressed in terms of the (adimensional) spin polarization $\zeta$ and the Wigner-Seitz parameter $r_{s}, r_{s}$ being the radius of an effective sphere including a single electron, $1 / n=4 \pi r_{s}^{3} / 3$.

\section{B. Variable mass}

In multishell QDs and also in homogeneous QDs embedded in a weak confining medium (allowing the electronic density to penetrate in the surroundings), the electron experiences different masses in different materials. We may say, alternatively, that the effective electron mass $m^{*}(\mathbf{r})$ has a multisteplike profile.

For a position-dependent mass, the appropriate Hermitian kinetic energy operator is given by ${ }^{30}$

$$
\hat{T}=-\frac{\hbar^{2}}{2} \nabla\left[\frac{1}{m(\mathbf{r})} \nabla\right] \text {. }
$$

The corresponding functional in the Kohn-Sham formulation should then be

$$
\hat{T}_{s}[n]=\sum_{i}^{\text {occ }} \int d \mathbf{r} \psi_{i}(\mathbf{r})^{*} \nabla\left[-\frac{\hbar^{2}}{2 m(\mathbf{r})} \nabla \psi_{i}(\mathbf{r})\right] .
$$

For bounded systems, i.e., for systems with zero electronic density at the boundaries, it may be rewritten as

$$
\hat{T}_{s}[n]=\sum_{i}^{\text {occ }} \int d \mathbf{r} \frac{\hbar^{2}}{2 m(\mathbf{r})}\left|\nabla \psi_{i}(\mathbf{r})\right|^{2} .
$$

It is worth remembering that the effective mass model, used to describe the electronic structure of QDs, integrates the microscopic details of the QD building block material lattice. Therefore, one cannot go into details of this unit cell with the model. This means, in particular, that the abrupt change of mass occurring when going from the QD to the surrounding matrix, or from shell to shell in a multishell QD, should be understood in a weak sense (an abrupt mismatch at the mathematical surface separating two materials may be a too severe imposition). Indeed, it is known that a dielectric mismatch so severe leads to a divergence in the single-particle self-polarization potential. This divergence produces a pathology in the Schrödinger equation not allowing it to be integrable. $^{31}$ In order to bypass this drawback, the abrupt mismatch is replaced by a continuous variation of the dielectric constant within a thin layer at the interface with a thickness down to a lattice constant. ${ }^{31-33}$ In this paper we assume this kind of smooth mismatch for all physical variables involved, namely, effective mass, dielectric constant, and confining potential (this last given by the band offset of the adjacent materials). 
As the effective mass does not come into Coulomb and exchange functionals, we do not consider these functionals for now. However, the correlation functional depends on the (dimensional) Wigner-Seitz radius $r_{s}$ and some scalings should be introduced, as we show next.

Correlation is an analytical functional $\epsilon_{c}\left(r_{s}, \zeta\right)$ written in atomic units, i.e., $\epsilon_{c}$ (hartree) and $r_{s}(\mathbf{r})$ (Bohr radius $a_{0}$ ), $\zeta$ being adimensional. A free mass $m_{0}$ is implicit for electrons. We may use, though, this very same functional for electrons with masses other than $m_{0}$ if we employ effective atomic units throughout. In such a case, the functional, as it is, will yield correlation energy in effective hartree if we inject $r_{s}$ in effective Bohr radii. In order to get back true atomic units, we should multiply $\epsilon_{c}$ by $m^{*} / \varepsilon^{2}$, where $m^{*}=m / m_{0}$ is the effective mass of the electron and $\varepsilon$ is the dielectric constant. ${ }^{34}$

This simple reasoning gives us the key to deal with variable mass systems: we should use true atomic units throughout, just making sure, at each $\mathbf{r}$, that both $\epsilon_{c}$ and its argument $r_{s}$ are properly scaled by $m^{*}(\mathbf{r}) / \varepsilon(\mathbf{r})^{2}$ and $m^{*}(\mathbf{r}) / \varepsilon(\mathbf{r})$, respectively.

\section{Variable dielectric constant}

Dielectric constant comes into Coulomb, exchange, and correlation functionals. The classical Coulomb term in vacuum,

$$
\Phi(\mathbf{r})=\int d \mathbf{r}^{\prime} \frac{n\left(\mathbf{r}^{\prime}\right)}{\left|\mathbf{r}-\mathbf{r}^{\prime}\right|}
$$

should be replaced by the one coming from the integrations of the Poisson equation,

$$
\nabla[\varepsilon(\mathbf{r}) \nabla \Phi(\mathbf{r})]=-4 \pi n(\mathbf{r}) .
$$

More involved is the exchange, as it is nonclassical. It can be obtained, in a homogeneous medium, in terms of the spin density matrices $n_{\sigma}\left(\mathbf{r}_{1}, \mathbf{r}_{2}\right)$ as $^{22}$

$$
E_{x}\left(n_{+}, n_{-}\right)=\frac{1}{2} \iint d \mathbf{r}_{1} d \mathbf{r}_{2} \frac{1}{r_{12}}\left[\left|n_{+}\left(\mathbf{r}_{1}, \mathbf{r}_{2}\right)\right|^{2}+\left|n_{-}\left(\mathbf{r}_{1}, \mathbf{r}_{2}\right)\right|^{2}\right] .
$$

If the dielectric constant of the homogeneous medium $\varepsilon$ is not unity as in a vacuum, we should replace $r_{12}$ with $\varepsilon r_{12}$ in the above equation. Next, by assuming homogeneous electron gas, which allows us to write the density matrix in terms of plane waves, we may end up with Eq. (5) (it should be divided by $\varepsilon$ if this constant is not unity).

The question arises in case $\varepsilon$ changes from a point to another. In a first attempt one may just replace $\epsilon_{x}(n, \zeta)$ with $\epsilon_{x}(n, \zeta) / \varepsilon(\mathbf{r})$ in Eq. (6). However, it is not consistent with the Coulomb functional, since Eq. (12) is not equivalent to $\varepsilon(\mathbf{r}) \nabla^{2} \Phi(\mathbf{r})=-4 \pi n(\mathbf{r})$. However, we may define the scaling parameter

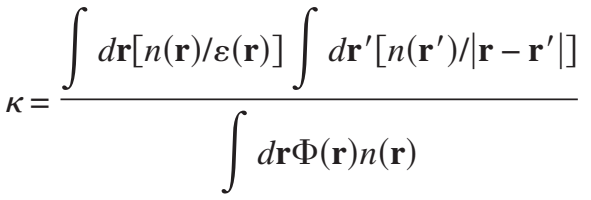

and an effective local dielectric constant $\bar{\varepsilon}(\mathbf{r})=\kappa \varepsilon(\mathbf{r})$ that allows to write the next identity,

$$
\int d \mathbf{r} \Phi(\mathbf{r}) n(\mathbf{r})=\iint d \mathbf{r} d \mathbf{r}^{\prime} \frac{n(\mathbf{r}) n\left(\mathbf{r}^{\prime}\right)}{\bar{\varepsilon}(\mathbf{r})\left|\mathbf{r}-\mathbf{r}^{\prime}\right|} .
$$

Therefore, scaling $\epsilon_{x}(n, \zeta)$ by $[\kappa \varepsilon(\mathbf{r})]^{-1}$ is globally consistent with the employed Coulomb functional. For the same reasons, we will replace $\varepsilon(\mathbf{r})$ by $[\kappa \varepsilon(\mathbf{r})]$ in the scaling factors coming into the correlation functional $\epsilon_{c}$.

The present extension of LSDA is, on one hand, local in the sense that the electronic density feels locally the physical properties of the medium, but, on the other hand, it is consistent with a rigorous calculation of Coulomb energy in an inhomogeneous medium.

\section{ILLUSTRATIVE CALCULATIONS}

As discussed in the previous section, if the permittivity is a constant, we may account for variable mass without introducing any approximation on top of LSDA (as far as the system is bounded, which will always be the case). Therefore, we devote this section to check the robustness of the proposed approach dealing with a variable permittivity. To this end, we have written a code for spherical quantum dots and calculated the very sensitive addition energies of electrons and holes in InAs and CdSe nanocrystals embedded in media with different dielectric constants. Our benchmark to compare with is a set of full configuration interaction (FCI) calculations carried out by Orlandi et al. ${ }^{18}$ The calculations employ an orbital basis set including $1 s, 1 p, 1 d$, and $2 s$ orbitals $^{35}$ and are exact up to seven particles. Larger QD populations up to 20 particles are calculated within a Hubbard-like approximation, i.e., only semidiagonal elements of the Hamiltonian are retained.

The present implementation of our approach for spherical multishell QDs uses a multistep function for the dielectric constant, for the confining potential, and for the effective mass, where, as discussed above, the abrupt mismatches are replaced by continuous variations within an extremely thin layer at the interface with a thickness down to a lattice constant. Mathematically, we do it by adding a set of Fermi functions as follows:

$$
\begin{aligned}
X(r)= & \sum_{l=1}^{n \text { shell }} X(l)\left(1-\frac{1}{1+\exp \left\{\left[r-R_{i}(l)\right] / a\right\}}\right) \\
& \times\left(\frac{1}{1+\exp \left\{\left[r-R_{f}(l)\right] / a\right\}}\right),
\end{aligned}
$$

where $X(r)$ is either the multistep dielectric constant $\varepsilon(r)$, the confining potential $V_{\text {conf }}(r)$, or the effective mass $m^{*}(r), R_{i}$ and $R_{f}$ are the initial and final shell radii, and $a$ the corresponding interface thickness. 

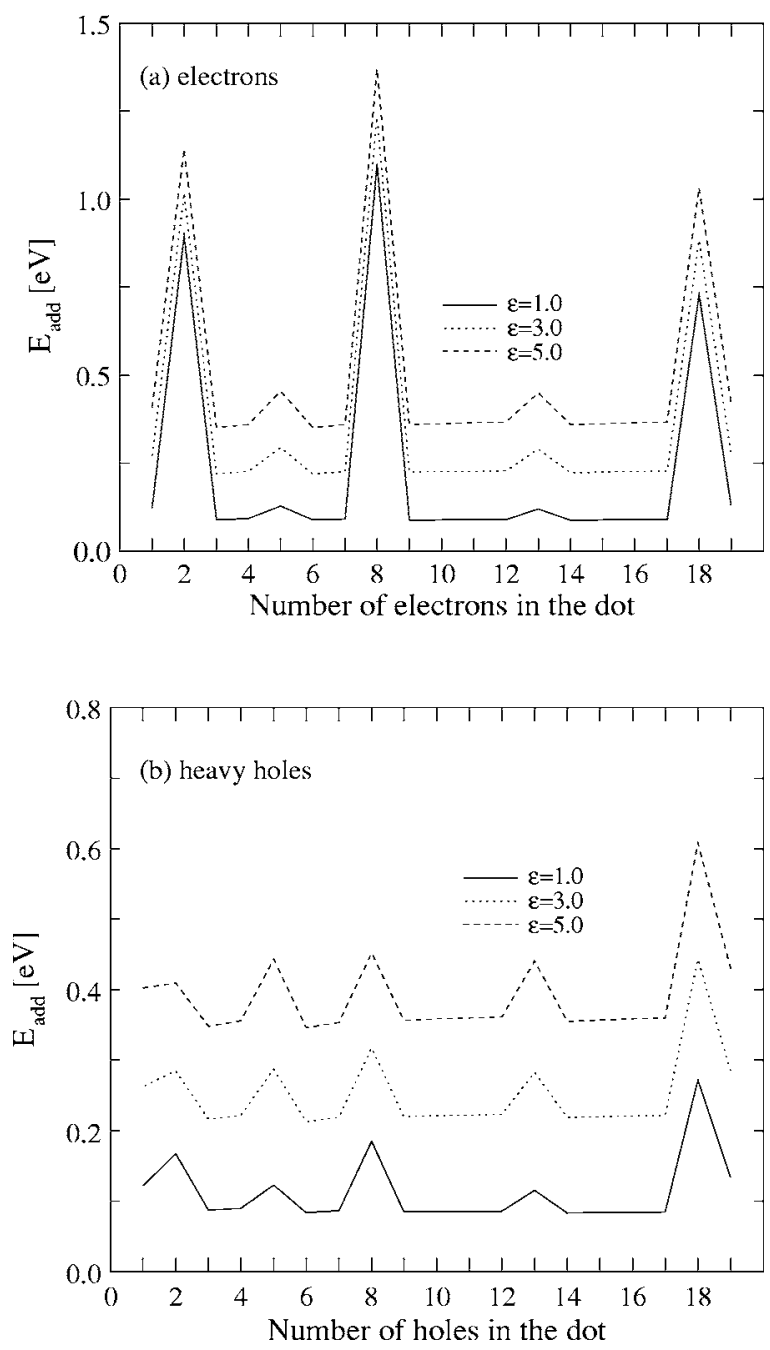

FIG. 1. Calculated addition energies (a) for electrons and (b) for heavy holes as a function of the number $N$ of particles in the dot (addition spectrum). Results are shown for a CdSe QD. Each curve corresponds to a different value of $\varepsilon=\varepsilon_{\mathrm{QD}} / \varepsilon_{\mathrm{ex}}$.

Kohn-Sham [Eq. (1)] and Poisson [Eq. (12)] equations have been solved numerically using the finite-difference method on the one-dimensional grid in spherical coordinates. The discretization scheme on a grid, extended far beyond the QD radius, yields either eigenvalue problems (Kohn-Sham) or linear systems of equations (Poisson) of asymmetric huge and sparse matrices that have been solved by standard matrix methods. The scaling parameter $\kappa$ is self-consistently calculated. The iterative process ends when complete selfconsistency is achieved.

The geometries and parameters employed in our calculations are those of Ref. 18, namely, a $2 \mathrm{~nm}$ radius CdSe QD $\left(m_{e}^{*}=0.11\right.$ for electrons, $m_{h}^{*}=1$ for holes, and $\left.\varepsilon_{\mathrm{QD}}=10\right)$ and a $3.2 \mathrm{~nm}$ InAs QD $\left(m_{e}^{*}=0.023\right.$ for electrons, $m_{h}^{*}=0.41$ for holes, and $\varepsilon_{\mathrm{QD}}=10.9$ ); both QDs are subjected to an infinite confinement and embedded in three different dielectric media defined by $\varepsilon=\varepsilon_{\mathrm{QD}} / \varepsilon_{\mathrm{ex}}=1,3,5$.

The results for addition energies of electrons in CdSe/InAs QDs are represented in Figs. 1(a) and 2(a) and should be compared with Figs. 1(a) and 2(a) of Ref. 18. As we can see, both qualitative and quantitative agreements are extremely good. The same agreement is achieved for addi-
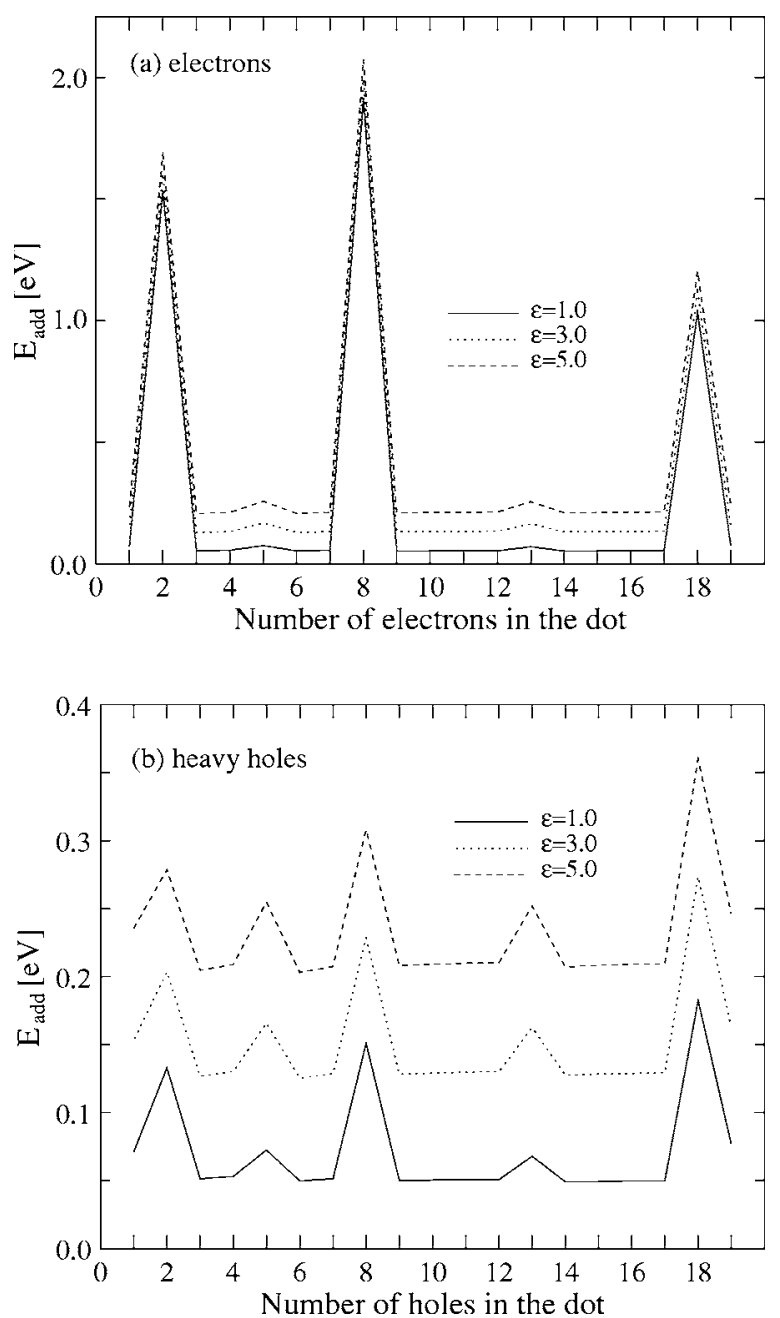

FIG. 2. Calculated addition energies (a) for electrons and (b) for heavy holes as a function of the number $N$ of particles in the dot (addition spectrum). Results are shown for an InAs QD. Each curve corresponds to a different value of $\varepsilon=\varepsilon_{\mathrm{QD}} / \varepsilon_{\mathrm{ex}}$.

tion energies of holes in InAs QD, Fig. 2(b), which should be compared with its partner in Ref. 18. Finally, addition energies of holes in CdSe QD are represented in Fig. 1(b) (to be compared with its partner in Ref. 18). The agreement is perfect if we allow the configurations that can be inferred from Ref. 18 [see Fig. 1(b)] to converge. However, a full research of the absolute energy minimum leads us to find out, just in this case of holes in CdSe, a few reconstructions. All of them

TABLE I. Disagreements between CI and LSDA: Holes in CdSe QD. The dominant CI configuration, the most stable LSDA configuration, and the relative energy spacing between these two configurations at LSDA level are shown.

\begin{tabular}{lll}
\hline \hline CI configuration & LSDA configuration & $\Delta E \quad(\%)$ \\
\hline$\varepsilon=3$ & $1 s 1 p^{3}$ & 1.0 \\
$1 s^{2} 1 p^{2}$ & & \\
$\varepsilon=5$ & $1 s 1 p^{3}$ & 1.7 \\
$1 s^{2} 1 p^{2}$ & $1 s 1 p^{3} 1 d^{5}$ & 0.2 \\
$1 s^{2} 1 p^{6} 1 d$ & $1 s^{2} 1 p^{3} 1 d^{5}$ & 0.1 \\
$1 s^{2} 1 p^{6} 1 d^{2}$ & $1 s^{2} 1 p^{4} 1 d^{5}$ & 0.01 \\
$1 s^{2} 1 p^{6} 1 d^{3}$ & & \\
\hline \hline
\end{tabular}




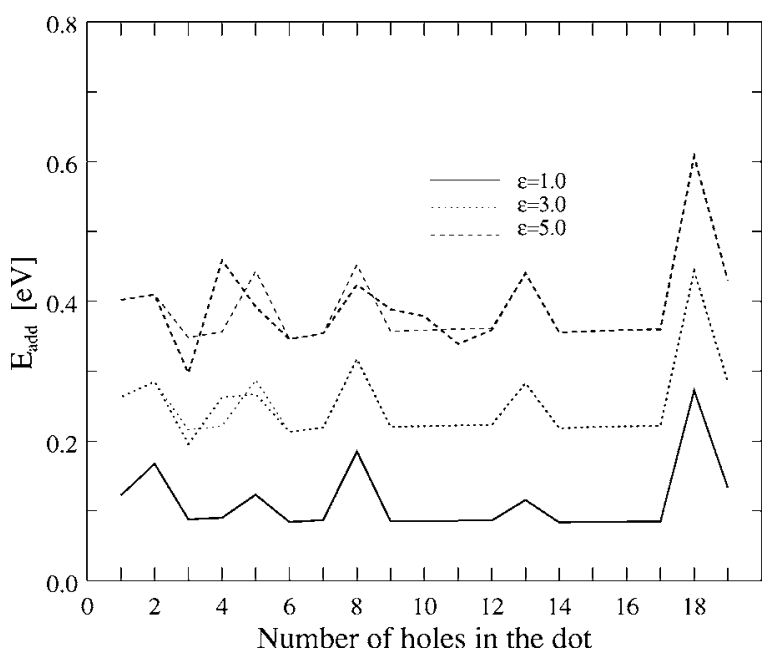

FIG. 3. Comparison of addition spectra for heavy holes in CdSe QD as a function of the number $N$ of particles in the dot, computed using LSDA absolute energy minimum configurations (thick lines) and dominant $\mathrm{CI}$ configurations (thin lines). Each curve corresponds to a different value of $\varepsilon$ $=\varepsilon_{\mathrm{QD}} / \varepsilon_{\mathrm{ex}}$. For $\varepsilon=1$ both configurations come to be identical.

are summarized in Table I, and the comparison of addition energies corresponding to both hole sequential fillings can be seen in Fig. 3. As we can see, the agreement with Ref. 18 is absolute also for holes in $\mathrm{CdSe}$ and $\varepsilon=1$. For $\varepsilon=3$ we find out a single reconstruction, the fourth hole comes into a $1 s 1 p^{3}$ configuration instead of a $1 s^{2} 1 p^{2}$. Our calculations show that the $1 s 1 p^{3}$ configuration is (just) $20 \mathrm{meV}$ more stable than $1 s^{2} 1 p^{2}$ (less than $1 \%$ of the total energy). It is worth stressing that holes in CdSe have an effective mass $m_{h}^{*}=1$. Therefore, the kinetic energy is small and the orbital levels turn out to be quite close, so that correlation plays a crucial role. On the other hand it is known that in high correlation regime LSDA shows a tendency to stabilize high spin. Then, it is difficult to assign this small disagreement with exact FCI results either to the own LSDA or to the scaling approximation employed. Finally, for $\varepsilon=5$ we find four reconstructions. Again, as can be seen in Table I the energetic differences between configurations are small.

In summary, a consistent extension of LSDA to account for mass and dielectric mismatches is presented in this paper. We prove that the extension to account for variable effective mass is exact. Some illustrative comparisons with more sophisticated CI calculations available show that our approach is also very reliable accounting for dielectric mismatches. Finally, it is worth stressing that the proposed extension of LSDA is extremely fast and computationally very low demanding (just as much as LSDA) in comparison with sophisticated CI calculations, so that one may deal with large many-particle system in inhomogeneous media without much effort.

\section{ACKNOWLEDGMENTS}

Continuous support from MEC-DGI Project Nos. CTQ2004-02315/BQU and FIS2005-01414, UJI-Bancaixa Project No. P1-B2002-01, and Generalitat de Catalunya 2005SGR00343 is gratefully acknowledged.
${ }^{1}$ R. C. Ashoori, Nature (London) 379, 413 (1996)

${ }^{2}$ L. Jacak, P. Hawrylak, and A. Wójs, Quantum Dots (Springer, Berlin, 1998).

${ }^{3}$ B. De Salvo, G. Ghibaudo, G. Pananakakis, P. Masson, T. Baron, N. Buffet, A. Fernandes, and B. Guillaumot, IEEE Trans. Electron Devices 48, 1789 (2001).

${ }^{4}$ D. V. Averin and K. K. Likhraev, in Mesoscopic Phenomena in Solids, edited by B. L. Altshuler, P. A. Lee, and R. A. Webb (North-Holland, Amsterdam, 1991).

${ }^{5}$ R. C. Ashoori, H. L. Störmer, J. S. Weiner, L. N. Pfeiffer, S. J. Pearton, K. W. Baldwin, and K. W. West, Phys. Rev. Lett. 68, 3088 (1992); R. C. Ashoori, H. L. Störmer, J. S. Weiner, L. N. Pfeiffer, K. W. Baldwin, and K. W. West, ibid. 71, 613 (1993).

${ }^{6}$ S. Tarucha, D. G. Austing, T. Honda, R. J. van der Hage, and L. P. Kouwenhoven, Phys. Rev. Lett. 77, 3613 (1996).

${ }^{7}$ J. Kainz, S. A. Mikhailov, A. Wensauer, and U. Rössler, Phys. Rev. B 65, 115305 (2002)

${ }^{8}$ S. M. Reimann and M. Manninen, Rev. Mod. Phys. 74, 1283 (2002).

${ }^{9}$ M. Pi, D. G. Austing, R. Mayol, K. Muraki, S. Sasaki, H. Tamura, and S. Tarucha, in Trends in Quantum Dots Research, edited by P. A. Ling (Nova Science, New York, 2005); D. G. Austing et al., in Nano-Physics and Bio-Electronics: A New Odyssey, edited by T. Chakraborty, F. Peeters, and U. Sivan (Elsevier, New York, 2002).

${ }^{10}$ H. Ow, D. R. Larson, M. Srivastava, B. A. Baird, W. W. Webb, and U. Wiesner, Nano Lett. 5, 113 (2005); M. Darbandi, R. Thomann, and T. Nann, Chem. Mater. 17, 5720 (2005); A. Mews, A. V. Kadavanich, U. Banin, and A. P. Alivisatos, Phys. Rev. B 53, R13242 (1996); D. Schooss, A. Mews, A. Eychmüller, and H. Weller, ibid. 49, 17072 (1994); A. Mews, A. Eychmüller, M. Giersig, D. Schooss, and H. Weller, J. Phys. Chem. 98, 934 (1994).

${ }^{11}$ G. W. Bryant and W. Jaskólski, Phys. Status Solidi B 224, 751 (2001); J. Planelles, J. G. Díaz, and J. Climente, Phys. Rev. B 65, 245302 (2002); J. Planelles, J. Climente, J. Díaz, and W. Jaskólski, J. Phys.: Condens. Matter 14, 12537 (2002); J. Climente, J. Planelles, J. Díaz, W. Jaskólski, and I. Aliaga, ibid. 15, 3593 (2003).

${ }^{12}$ U. Woggon, Optical Properties of Semiconductor Quantum Dots (Springer, Berlin, 1996).

${ }^{13}$ See, e.g., M. Bruchez Jr., M. Moronne, P. Gin, S. Weiss, and A. P. Alivisatos, Science 281, 2013 (1998); W. C. W. Chan and S. Nie, ibid. 281, 2016 (1998).

${ }^{14}$ A. P. Alivisatos, Science 271, 933 (1996); MRS Bull. 23, 18 (1998).

${ }^{15}$ U. Banin, Y. W. Cao, D. Katz, and O. Millo, Nature (London) 400, 542 (1999).

${ }^{16}$ B. Alperson, I. Rubinstein, and G. Hodes, Appl. Phys. Lett. 75, 1751 (1999).

${ }^{17}$ D. L. Klein, R. Roth, A. K. L. Lim, A. P. Alivisatos, and P. L. Mceuen, Nature (London) 389, 699 (1997).

${ }^{18}$ A. Orlandi, M. Rontani, G. Goldoni, F. Manghi, and E. Molinari, Phys. Rev. B 63, 045310 (2001).

${ }^{19}$ A. Orlandi, G. Goldoni, F. Manghi, and E. Molinari, Semicond. Sci. Technol. 17, 1302 (2002).

${ }^{20}$ J. Sée, P. Dollfus, and S. Galdin, J. Appl. Phys. 92, 3141 (2002).

${ }^{21}$ M. Şahin and M. Tomak, Phys. Rev. B 72, 125323 (2005); Physica E (Amsterdam) 28, 247 (2005).

${ }^{22}$ R. G. Parr and W. Yang, Density-Functional Theory of Atoms and Molecules (Oxford University Press, Oxford, 1989).

${ }^{23}$ E. Lipparini, Modern Many-Particle Physics (World Scientific, Hackensack, NJ, 2003).

${ }^{24}$ W. Khon and L. J. Sham, Phys. Rev. 140, A1133 (1965).

${ }^{25}$ P. Hohenberg and W. Khon, Phys. Rev. 136, B864 (1964).

${ }^{26}$ M. Levy, Proc. Natl. Acad. Sci. U.S.A. 76, 6062 (1979).

${ }^{27}$ U. von Barth and L. Hedin, J. Phys. C 5, 1629 (1972).

${ }^{28}$ It should be emphasized that the Kohn-Sham equation [Eq. (1)] should be solved in a geometrically unrestricted scheme, i. e., the symmetry of the solution should not be constrained by the symmetry of the confinement. In such a case, symmetry broken ground states may be found for large values of the Wigner-Seitz parameter $r_{s}$ (strong correlation regime). However, in general, a symmetry restricted DFT scheme gives reliable approximations to the exact ground-state energies and densities.

${ }^{29}$ J. P. Perdew and A. Zunger, Phys. Rev. B 23, 5048 (1981).

${ }^{30}$ D. BenDaniel and C. B. Duke, Phys. Rev. 152, 683 (1966).

${ }^{31}$ L. Bányai and S. W. Koch, Semiconductor Quantum Dots (Word Scientific, Singapore, 1993). 
${ }^{32}$ F. Stern, Phys. Rev. B 17, 5009 (1978).

${ }^{33}$ P. G. Bolcatto and C. R. Proetto, J. Phys.: Condens. Matter 13, 319 (2001).

${ }^{34}$ Actually, one does not inject $r_{s}$ into $\epsilon_{c}$, but $n(\mathbf{r})$. In such a case, we should inject it in effective units, i.e., by scaling $n(\mathbf{r})$ (a.u.) by a factor $\left(\varepsilon / m^{*}\right)^{3}$.
${ }^{35}$ Using our approach we have seen that this basis set is appropriate for electrons in the two QDs studied. In the case of holes this basis set is large enough up to 18 holes. The 19th and 20th holes come then to fill $1 f$ instead of $2 s$. However, in order to carry out proper comparison, we have used the same basis set as in Ref. 18 . 